\title{
Deficiency of IRTKS as an adaptor of insulin receptor leads to insulin resistance
}

\author{
Li-Yu Huang ${ }^{1,2, *}$, Yu-Ping Wang ${ }^{1,2,7, *}$, Bao-Feng Wei ${ }^{1,2}$, Jian Yang ${ }^{3}$, Ji-Qiu Wang ${ }^{3}$, Bing-Hao Wu ${ }^{1,2}$, \\ Zhuang-Zhuang Zhang ${ }^{1,2}$, Ying-Yong Hou ${ }^{4}$, Wei-Ming Sun ${ }^{5}$, Ren-Ming Hu ${ }^{6}$, Guang Ning ${ }^{3}$, Ze-Guang Han ${ }^{1,2}$
}

${ }^{I}$ Key Laboratory of Systems Biomedicine (Ministry of Education) of Rui-Jin Hospital, Shanghai Jiao Tong University School of Medicine, 197 Rui-Jin 2nd Road, Shanghai 200025, China; ${ }^{2}$ Shanghai-MOST Key Laboratory for Disease and Health Genomics, Chinese National Human Genome Center at Shanghai, 351 Guo Shou-Jing Road, Shanghai 201203, China; ${ }^{3}$ Department of Endocrinology and Metabolism, Rui-Jin Hospital, Shanghai Jiao-Tong University School of Medicine, 197 RuiJin 2nd Road, Shanghai 200025, China; ${ }^{4}$ Department of pathology, Zhongshan Hospital, Fudan University, Shanghai 200032, China; ${ }^{5}$ Department of Endocrinology, The First Hospital of Lanzhou University, 1 Dong-Gang West Road, Lanzhou, Gansu 730000, China; ${ }^{6}$ Department of Endocrinology and Metabolism, Huashan Hospital, Fudan University, Shanghai 200040, China; ${ }^{7}$ Department of Medical Biochemistry and Molecular Biology, School of Basic Medical Sciences, Lanzhou University, 199 DongGang West Road, Lanzhou, Gansu 730000, China

IRTKS encodes a member of the IRSp53/MIM homology domain family, which has been shown to play an important role in the formation of plasma membrane protrusions. Although the phosphorylation of IRTKS occurs in response to insulin stimulation, the role of this protein in insulin signaling remains unknown. Here we show that IRTKS-deficient mice exhibit insulin resistance, including hyperglycemia, hyperinsulinemia, glucose intolerance, decreased insulin sensitivity, and increased hepatic glucose production. The administration of ectopic IRTKS can ameliorate the insulin resistance of IRTKS-deficient and diabetic mice. In parallel, the expression level of IRTKS was significantly decreased in diabetic mouse model. Furthermore, DNA hypermethylation of the IRTKS promoter was also observed in these subjects. We also show that IRTKS, as an adaptor of the insulin receptor (IR), modulates IR-IRS1PI3K-AKT signaling via regulating the phosphorylation of IR. These findings add new insights into our understanding of insulin signaling and resistance.

Keywords: insulin resistance; IRTKS; insulin receptor

Cell Research (2013) 23:1310-1321. doi:10.1038/cr.2013.99; published online 30 July 2013

\section{Introduction}

Human Insulin Receptor Tyrosine Kinase Substrate (IRTKS), also known as BAI1-associated protein 2-like 1 (BAIAP2L1), was previously cloned from endocrine organs in our laboratory (GenBank accession number: AF119666) [1] and encodes a member of the IRSp53/ MIM (missing-in-metastasis) homology domain fami-

*These two authors contributed equally to this work.

Correspondence: Ze-Guang Han

Tel: +86-21-50801325; Fax: +86-21-50800402

Email: hanzg@chgc.sh.cn

Received 17 February 2013; revised 13 June 2013; accepted 26 June 2013; published online 30 July 2013 ly. Members of this family have been shown to play an important role in the formation of plasma membrane protrusions [2]. IRSp53-like proteins contain a conserved IRSp53/MIM homology domain (IMD) at the N-terminus and a canonical SH3 domain near the C-terminus [3]. The IMD belongs to the larger family of Bin-amphyipysin-Rvs67 (BAR) domains, which can bundle actin filaments and induce membrane protrusions $[4,5]$. IRSp53, which is the founding member of the IRSp53/ MIM homology domain family, was originally identified as a phosphorylation substrate for the insulin receptor tyrosine kinase and is tyrosine-phosphorylated in response to stimulation by insulin $[6,7]$. However, previous research focused primarily on its role in regulating the actin cytoskeleton and the formation of membrane 
protrusions including lamellipodia and filopodia in motile cells [2-5] and not on its role in insulin signaling. It was recently reported that IRSp53-knockout (KO) mice do not exhibit morphological abnormalities but do show learning and memory deficits in the contextual fear-conditioning paradigm $[8,9]$. However, whether IRSp53 deficiency disrupts insulin signaling and leads to insulin resistance was not evaluated.

Similarly to IRSp53, IRTKS can induce the clustering of short actin bundles, cause membrane protrusions [10] and trigger pathogen-driven actin assembly [11-13]. Tyrosine phosphorylation of IRTKS occurred in response to insulin stimulation when ectopic IRTKS and insulin receptor (IR) $\beta$-subunit (IR $\beta$ ) were co-transfected into COS-7 cells [10], which implies that IRTKS could be a component of insulin signaling. However, the molecular mechanism by which IRTKS contributes to insulin signaling is unknown. We noted that unlike IRSp53 expression, which is relatively enriched in the brain, IRTKS expression is widespread and is particularly found in the liver [10], raising the possibility that IRTKS might play an important role in liver. To explore the contribution of IRTKS to insulin signaling, insulin resistance and diabetes development, we generated IRTKS-deficient mice by targeting the gene via homologous recombination.

\section{Results}

IRTKS deficiency leads to insulin resistance

To determine whether IRTKS is involved in insulin signaling and resistance, we first generated IRTKS-KO mice by disrupting the first exon containing the start codon of the IRTKS gene (Figure 1A and Supplementary information, Figure S1). The tissue expression pattern of IRTKS, as detected by RT-PCR (data not shown) and western blotting, showed that in homozygous KO mice, IRTKS expression was completely undetectable, whereas in heterozygous (HET) mice, its expression was obviously decreased (Figure 1B and 1C). These results indicate the successful development of a genetically engineered IRTKS-deficient mouse model.

The IRTKS-KO mice fed a regular diet did not show abnormal characteristics in growth and fertility. The body weight of IRTKS-KO mice was also similar to those of wild-type (WT) littermates under chow diet (Supplementary information, Figure S2A and S2B). We monitored blood glucose levels and found that blood glucose levels were significantly elevated in the fasting male IRTKS-KO mice (Figure 2A). Female IRTKS-KO mice also showed a slightly but significantly elevated blood glucose level (Supplementary information, Figure S2C). In parallel,

A

Wild-type allele

Targeting Vector

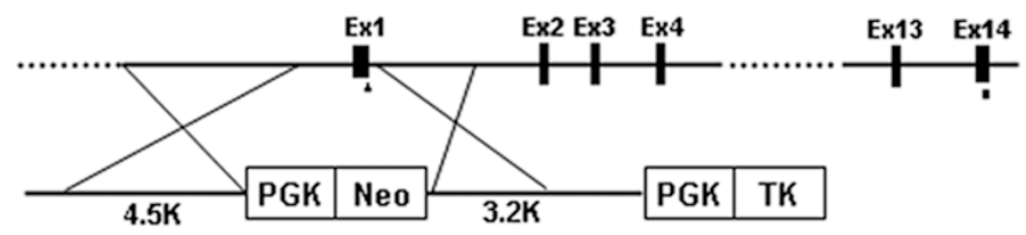

Targeted allele

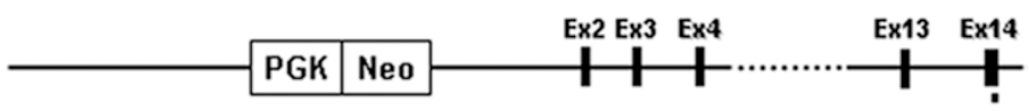

A, ATG; $=$, TGA;

B
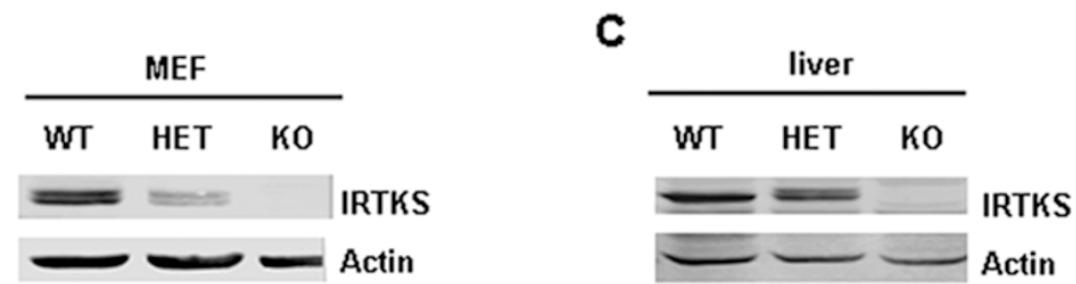

Figure 1 Construction and identification of IRTKS-KO mice. (A) Schematic diagram for the construction of IRTKS KO mice via targeting the first exon of IRTKS. (B, C) IRTKS expression levels in MEF cells (B) and livers (C) from IRTKS -KO, HET and WT mice. Actin was used as loading control. 
A

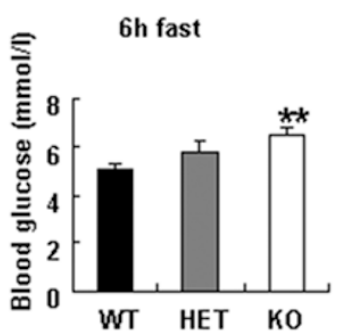

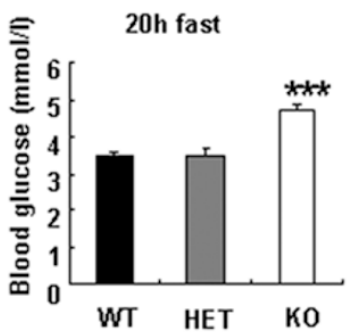

B

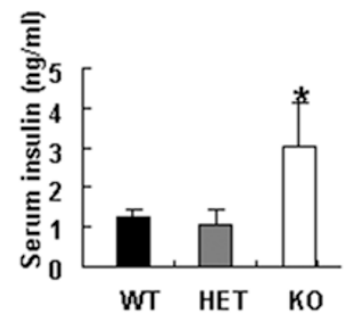

C

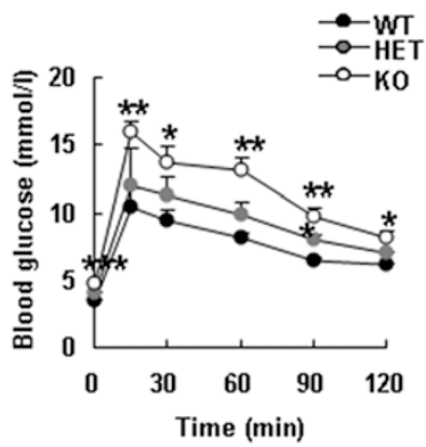

D

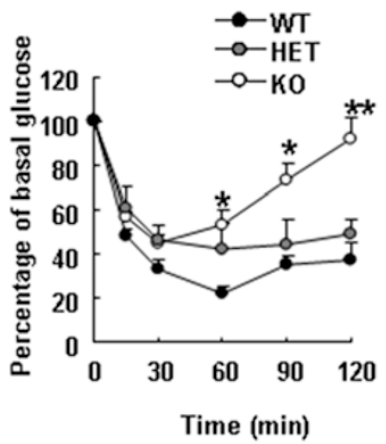

E

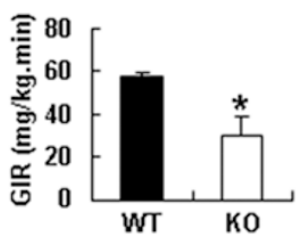

$\mathbf{F}$

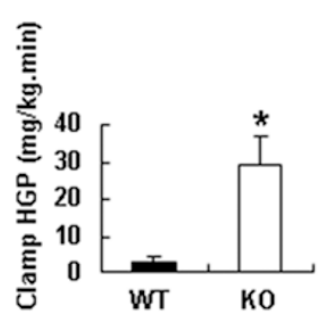

G

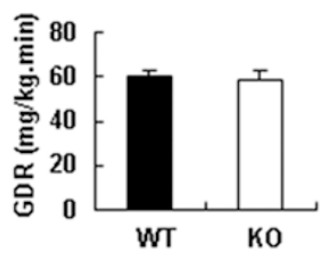

H

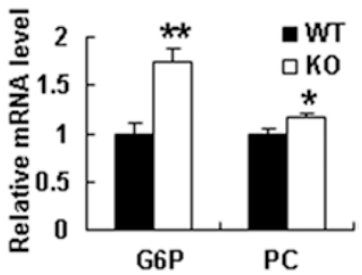

Figure 2 IRTKS deficiency leads to insulin resistance. (A) Blood glucose levels in 3.5-month-old male IRTKS-KO, HET mice and WT littermates $(n=5)$. (B) Serum insulin levels in 3.5-month-old male IRTKS-KO, HET mice and WT littermates $(n=$ 5-8). (C) Glucose tolerance test (GTT) in IRTKS-KO, HET mice and WT littermates $(n=5-8)$ (fasted overnight and then ip glucose $(1.5 \mathrm{~g} / \mathrm{kg}$ )). (D) Insulin tolerance test (ITT) (ip insulin (1 U/kg)) $(n=5-8)$. (E-G) In hyperinsulinemic-euglycemic clamp studies, glucose infusion rate (GIR) (E), clamped hepatic glucose production (HGP) (F) and glucose disappearance rate (GDR) (G) were evaluated in 5-month-old male IRTKS-KO mice and WT littermates $(n=4)$. (H) Relative mRNA levels of glucose-6-phosphatase (G6P) and pyruvate carboxylase (PC) in IRTKS-KO mice and WT littermates $(n=4)$. Data are presented as mean \pm sem. ${ }^{*} P<0.05,{ }^{* *} P<0.01,{ }^{* *} P<0.001$ versus control.

serum insulin level significantly increased in IRTKSKO mice (Figure 2B). To evaluate the effect of IRTKS on insulin resistance, we performed glucose tolerance test (GTT) and insulin tolerance test (ITT) on IRTKS$\mathrm{KO}$ mice. These tests revealed glucose intolerance and the decreased insulin sensitivity in IRTKS-KO and HET mice as compared with WT littermates (Figure 2C and 2D, Supplementary information, Figure S2D and S2E).

To further evaluate the role of IRTKS in modulating whole-body insulin sensitivity, we performed hyperinsulinemic-euglycemic clamp studies on 5-month-old IRTKS-KO mice. The glucose infusion rate (GIR) was significantly reduced in male and female IRTKS-KO mice (Figure 2E, Supplementary information, Figure S2F) whereas the clamped hepatic glucose production (HGP) was obviously higher in IRTKS-KO mice than in WT littermates (Figure 2F). These data demonstrate that insulin sensitivity is severely impaired in IRTKS-deficient mice and that the resultant utilization of blood glucose by the liver is attenuated by insulin resistance. Meanwhile, the glucose disappearance rate (GDR) of IRTKS-KO mice was not reduced compared with WT littermates (Figure $2 \mathrm{G})$, implying that insulin resistance may not occur in muscles, possibly due to the low expression of IRTKS 
in skeletal muscles (Supplementary information, Figure S2G) [10].

We also evaluated the expression of certain genes involved in gluconeogenesis in livers from IRTKS-deficient mice by real-time RT-PCR. Gluconeogenesis-associated genes such as glucose-6-phosphatase (G6P) and pyruvate carboxylase $(\mathrm{PC})$ were upregulated, indicating that hepatic gluconeogenesis increases in IRTKS-KO mice (Figure 2H).

\section{Ectopic IRTKS can reverse insulin resistance}

To further define the effect of IRTKS on insulin sensitivity and glucose homeostasis, we performed a rescue experiment by intravenously injecting a recombinant adenovirus vector containing IRTKS (Ad-IRTKS) into $I R T K S-\mathrm{KO}$ mice. As expected, upon injection of ectopic $I R T K S$, the blood glucose levels of IRTKS-KO mice significantly decreased as compared to that of those injected with empty vector (Figure 3A); and the increased glucose tolerance and insulin sensitivity were confirmed by the
GTT and ITT tests (Figure 3B and 3C), where both GTT and ITT curves derived from IRTKS-KO mice injected with Ad-IRTKS were comparable to those from WT littermates. In addition, we injected an Ad-IRTKS into WT mice, and analyzed the glucose tolerance and insulin sensitivity of these mice. The data revealed that the glucose tolerance and insulin sensitivity in WT mice with ectopic IRTKS was significantly increased as compared to that of WT control with empty vector (Figure 3D and $3 \mathrm{E})$. In this experiment, the food intake and body weight of both groups showed no significant difference (data not shown).

We further evaluated whether ectopic IRTKS can attenuate insulin resistance in $d b / d b$ mice, which represent a well-established diabetic insulin resistance mouse model. Surprisingly, the administration of Ad-IRTKS significantly reduced blood glucose levels in diabetic $d b$ / $d b$ mice (Figure $3 \mathrm{~F}$ ) and ameliorated glucose intolerance, as shown by the GTT test (Figure 3G), although the GTT curve was not comparable to that of WT (lean) mice.
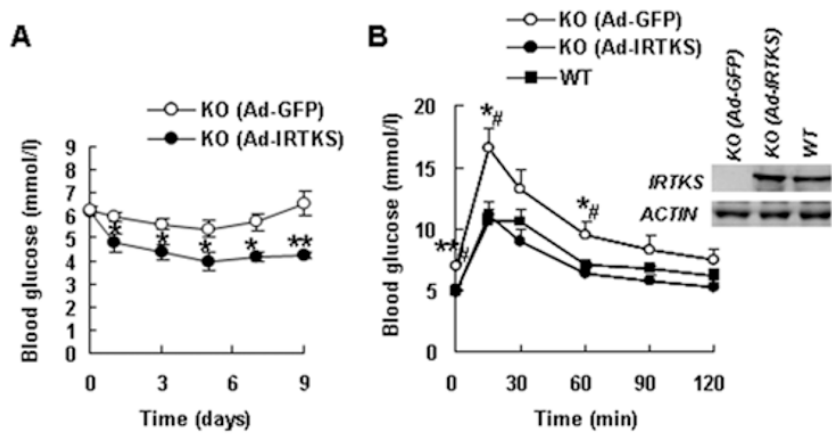

E

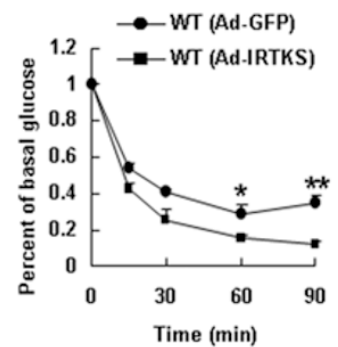

$\mathbf{F}$

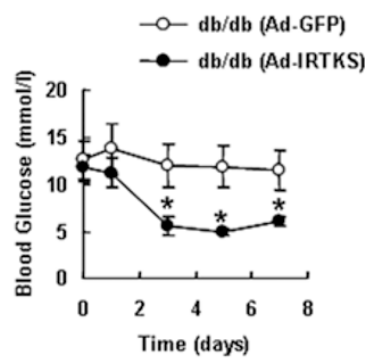

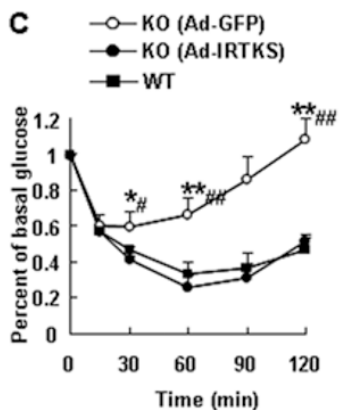
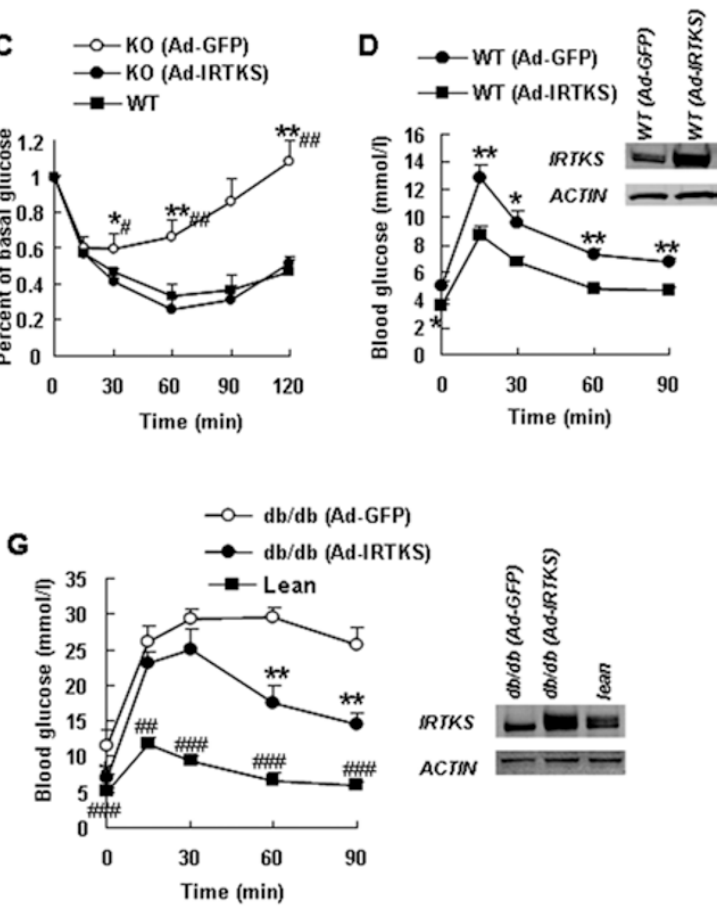

Figure 3 Ectopic IRTKS reverses insulin resistance in IRTKS-KO mice and $d b / d b$ mice. (A) Blood glucose levels of IRTKSKO mice (5-month-old) injected with Ad-GFP or Ad-IRTKS $(n=5)$ under a $6 \mathrm{~h}$ fast stage. (B, C) Glucose tolerance test (GTT) (fasted $6 \mathrm{~h}$ and then ip glucose $1.5 \mathrm{~g} / \mathrm{kg}$ ) (B) and insulin tolerance test (ITT) (ip insulin $1 \mathrm{U} / \mathrm{kg}$ ) (C) in IRTKS-KO mice injected with Ad-GFP or Ad-IRTKS $(n=5)$. WT as control $(n=5)$. (D, E) GTT (fasted $6 \mathrm{~h}$ and then ip glucose $1.5 \mathrm{~g} / \mathrm{kg}$ ) (D) and ITT (ip insulin $1 \mathrm{U} / \mathrm{kg}$ ) (E) in 5-month-old WT male mice injected with Ad-GFP or Ad-IRTKS $(n=5)$. (F) Blood glucose levels of $d b /$ $d b$ mice injected with Ad-GFP or Ad-IRTKS ( $n=4)$ under a $6 \mathrm{~h}$ fast stage. (G) GTT (fasted $6 \mathrm{~h}$ and then ip glucose $1.5 \mathrm{~g} / \mathrm{kg}$ ) in $d b / d b$ mice injected with Ad-GFP or Ad-IRTKS $(n=4)$. Lean mice as control $(n=5)$. IRTKS protein expression in livers of mice after injection with adenovirus was shown by western blotting. Data are presented as mean \pm s.e.m. ${ }^{*} P<0.05$, ${ }^{* *} P<0.01$ versus Ad-IRTKS. ${ }^{\#} P<0.05,{ }^{\#} P<0.01,{ }^{\# \#} P<0.001$ versus WT or lean mice. 
Moreover, the insulin release stimulated by glucose was improved in $d b / d b$ mice with Ad-IRTKS (Supplementary information, Figure S3A and S3B), and insulin sensitivity was increased, as indicated by ITT test (Supplementary information, Figure S3C). In these tests, there was no significant difference in food intake and body weight between the two groups (data not shown).

These results suggest that IRTKS is required for the regulation of insulin sensitivity and glucose homeostasis and that the administration of IRTKS may possess therapeutic potential against insulin resistance.

\section{IRTKS modulates insulin signaling}

The above observations imply that IRTKS could be essential for insulin signaling. To test this hypothesis, we evaluated the tyrosine phosphorylation state of endogenous IRTKS in response to insulin stimulation by an immunoprecipitation assay. The data indicate that IRTKS is tyrosine-phosphorylated in the liver cancer cell line Huh-7 (Supplementary information, Figure S4A), which is consistent with previous studies in COS-7 cells co-transfected with ectopic IRTKS and IR $\beta$ [10]. These suggest that IRTKS could be a component of insulin signaling. Subsequently, we assessed the phosphorylation of certain key molecules of insulin signaling, including IR at tyrosine residues $1162 / 1163$ that are necessary for full kinase activity [14], AKT and glycogen synthase kinase $-3 \beta$ (GSK-3 $\beta$ ), in IRTKS-deficient mice. Interestingly,
A

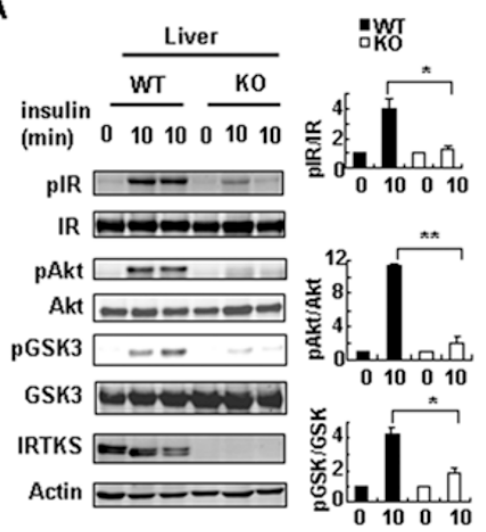

B
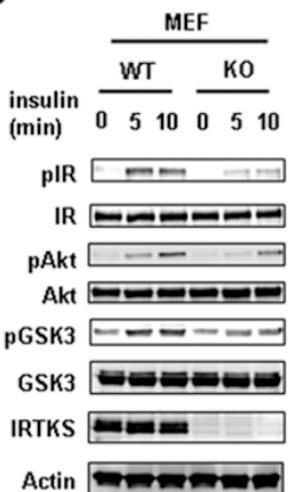

Actin meneren

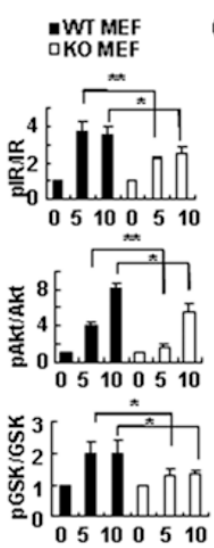

C

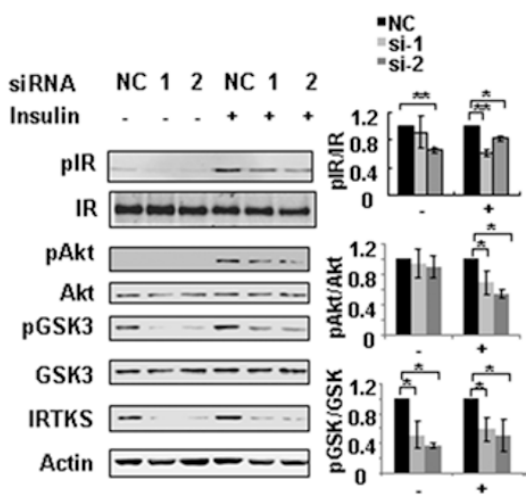

D

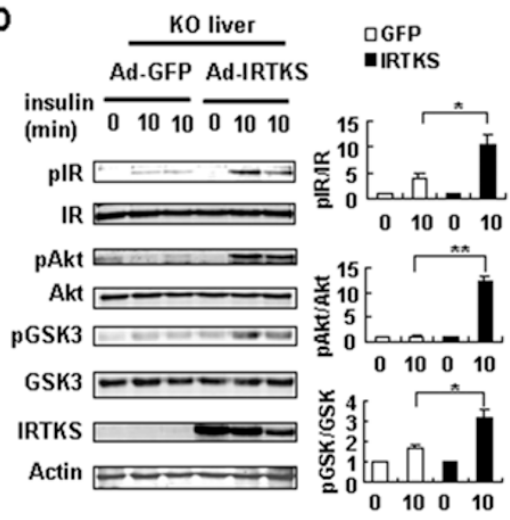

E

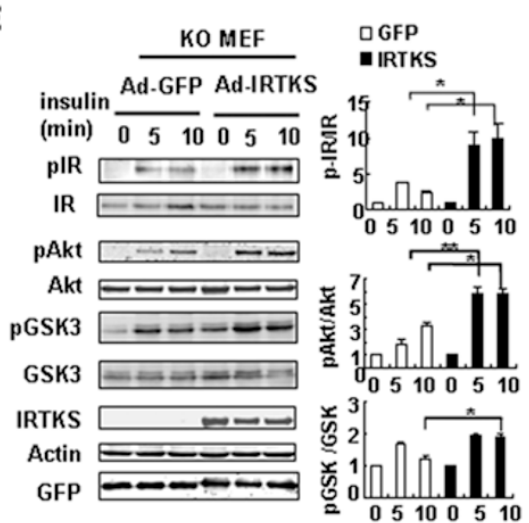

Figure 4 IRTKS regulates the insulin signaling pathway. (A-B) Phosphorylation levels of IR at tyrosine residues Tyr1162/1163, AKT at serine 473 and GSK-3 $\beta$ at serine 9 were assessed by western blotting assay in livers (A) and MEFs (B) from IRTKSKO mice and WT littermates. (C) Phosphorylation levels of IR at Tyr1162/1163, AKT at serine 473 and GSK-3 $\beta$ at serine 9 were evaluated in YY-8103 cells upon IRTKS knockdown. (D, E) Phosphorylation levels of the proteins in A-C were evaluated in livers (D) and MEF cells (E) from IRTKS-KO mice when infected with Ad-GFP or Ad-IRTKS. (A-E) Liver extracts were collected from mice fasted overnight and then injected with insulin $(1 \mathrm{U} / \mathrm{kg})$ for the indicated times. Cell lysates were extracted from MEFs and YY-8103 cells starved overnight and then stimulated with insulin $(4 \mu \mathrm{g} / \mathrm{ml})$ for the indicated times. Statistical analysis on these data was shown on the right. The phosphorylation levels of the proteins were evaluated based on intensity of their bands, where relative phosphorylation levels were normalized as 1 at the absence of insulin. All statistical analysis was calculated using Student's $t$-test. The data are mean $\pm \mathrm{sd}$. ${ }^{*} P<0.05$, ${ }^{* \star} P<0.01$ versus control. 
the phosphorylation of these molecules in response to insulin stimulation was significantly attenuated in the liver, adipose tissue and mouse embryo fibroblasts (MEFs) of IRTKS-KO mice (Figure 4A and 4B, Supplementary information, Figure S4B), suggesting that, in addition to liver, insulin resistance could also exist in other peripheral tissues including adipose tissue of these IRTKS-deficient mice. However, there was no obvious attenuation of insulin signaling in the muscle of IRTKS-KO mice (Supplementary information, Figure S4C), in consistent with the phenotype that GDR of IRTKS-KO mice shows no reduction in the above clamp test.

Our previous data showed that IRTKS could be important for ubiquitination and processing of $\mathrm{p} 53$ protein [15]. In this study, however, the protein levels of the insulin receptor and downstream molecules IRS $1 / 2$, AKT and GSK-3 $\beta$ were unchanged in tissues or MEFs from IRTKS-deficient mice (Figure 4A and 4B). We also analyzed the ubiquitination level of the insulin receptor in livers from WT and IRTKS-KO mice (Supplementary information, Figure S4D). The result indicated that the ubiquitination level of insulin receptor was similar between WT and IRTKS-KO mice, implying that IRTKS may not affect IR processing.

To confirm the effect of IRTKS on insulin signaling, we further evaluated these downstream molecules in human liver cancer cell lines by knockdown of endogenous IRTKS using small interfering RNA (siRNA). The data show that when IRTKS was knocked down, the phosphorylation levels of IR, AKT and GSK-3 $\beta$ significantly decreased in YY-8103 and Huh7 cells (Figure 4C, Supplementary information, Figure S4E). These data indicate that IRTKS deficiency can significantly impair insulin signaling.

To test whether ectopic IRTKS can reverse defective insulin signaling, we examined the phosphorylation of IR, AKT and GSK-3 $\beta$ in the liver of IRTKS-KO mice injected with Ad-IRTKS. The phosphorylation of these proteins was significantly enhanced upon injection of AdIRTKS compared with the injection of an empty Ad-GFP vector (Figure 4D). Moreover, we infected MEFs from $I R T K S$-KO mice with Ad-IRTKS. The phosphorylation of IR, AKT and GSK-3 $\beta$ in these cells also significantly increased upon ectopic expression of IRTKS (Figure 4E). Furthermore, we also transfected YY-8103 cells having endogenous IRTKS expression with the recombinant plasmid pCMV-IRTKS. Enhanced IR $\beta$ phosphorylation was observed in these cells (Supplementary information, Figure S4F). Here we also observed the increased phosphorylation level of ERK (Supplementary information, Figure S4F), which has been known to be activated by IR signaling. Taken together, the data support the posi- tive modulation of insulin signaling by IRTKS.

IRTKS encodes a member of the IRSp53/MIM homology domain family. To assess whether the effect of IRTKS on insulin signaling is specific, we employed the synthesized siRNA to knock down the highly homologous paralog IRSp53, the founder member of this family. The result showed that the knockdown of endogenous IRSp53 did not attenuate the phosphorylation levels of AKT and GSK-3 $\beta$ in YY-8103 and Huh-7 cells (Supplementary information, Figure S5A and S5B). The data implied that the effect of IRTKS on insulin signaling could be distinct from other members of this family.

\section{IRTKS is an adaptor of insulin receptor}

Upon the loss or gain of IRTKS, the phosphorylation of tyrosine $1162 / 1163$ of IR was significantly altered (Figure 4A-4E). We also noted that, in Huh-7 cells, GFPtagged IRTKS was localized near the cell membrane 10$30 \mathrm{~min}$ after insulin stimulation (Figure 5A); and the colocalization of ectopic IRTKS and IR was enhanced at 10 min after insulin stimulation (Supplementary information, Figure S6A). This implied that IRTKS may interact with IR and modulate IR-IRS1/2-PI3K-AKT signal transduction in response to insulin stimulation.

To test this hypothesis, we performed reciprocally endogenous co-immunoprecipitation (Co-IP) experiments in mouse liver with anti-IRTKS, -IR and -IRS1/2 antibodies. Not only IR, but also IRS1 and IRS2 were immunoprecipitated by an anti-IRTKS antibody in liver from WT with insulin stimulation, but not in IRTKS-KO mice (Figure 5B). Conversely, IRTKS was immunoprecipitated by anti-IR and -IRS1 antibodies in liver from WT mice after insulin stimulation (Figure 5C and 5D), whereas the phosphorylation levels of the immunoprecipitated IR (Tyr1162/1163) and IRS1 (Tyr612) as well as the association of IRS1 with p85, the key regulatory subunit of PI3K, were reduced in IRTKS-KO mice, in consistent with the above observation (Figure 4A, Supplementary information, Figure S6B). Similarly, the tyrosine phosphorylation of the immunoprecipitated IRS2 was also decreased in IRTKS-KO mice (Supplementary information, Figure $\mathrm{S} 6 \mathrm{C}$ ). Interestingly, the ectopic IRTKS injected into IRTKS-KO mice was also immunoprecipitated by anti-IR and -IRS1 antibodies in liver, along with increased phosphorylation level of immunoprecipitated IRS1 (Figure 5E). Moreover, we employed the same immunoprecipitation assay to investigate the association between IRTKS and IR in human Huh-7 cells, and we also observed the interaction (Supplementary information, Figure S6D). Furthermore, we performed GST pulldown assays with in vivo and in vitro translated protein products to determine whether IRTKS can directly in- 


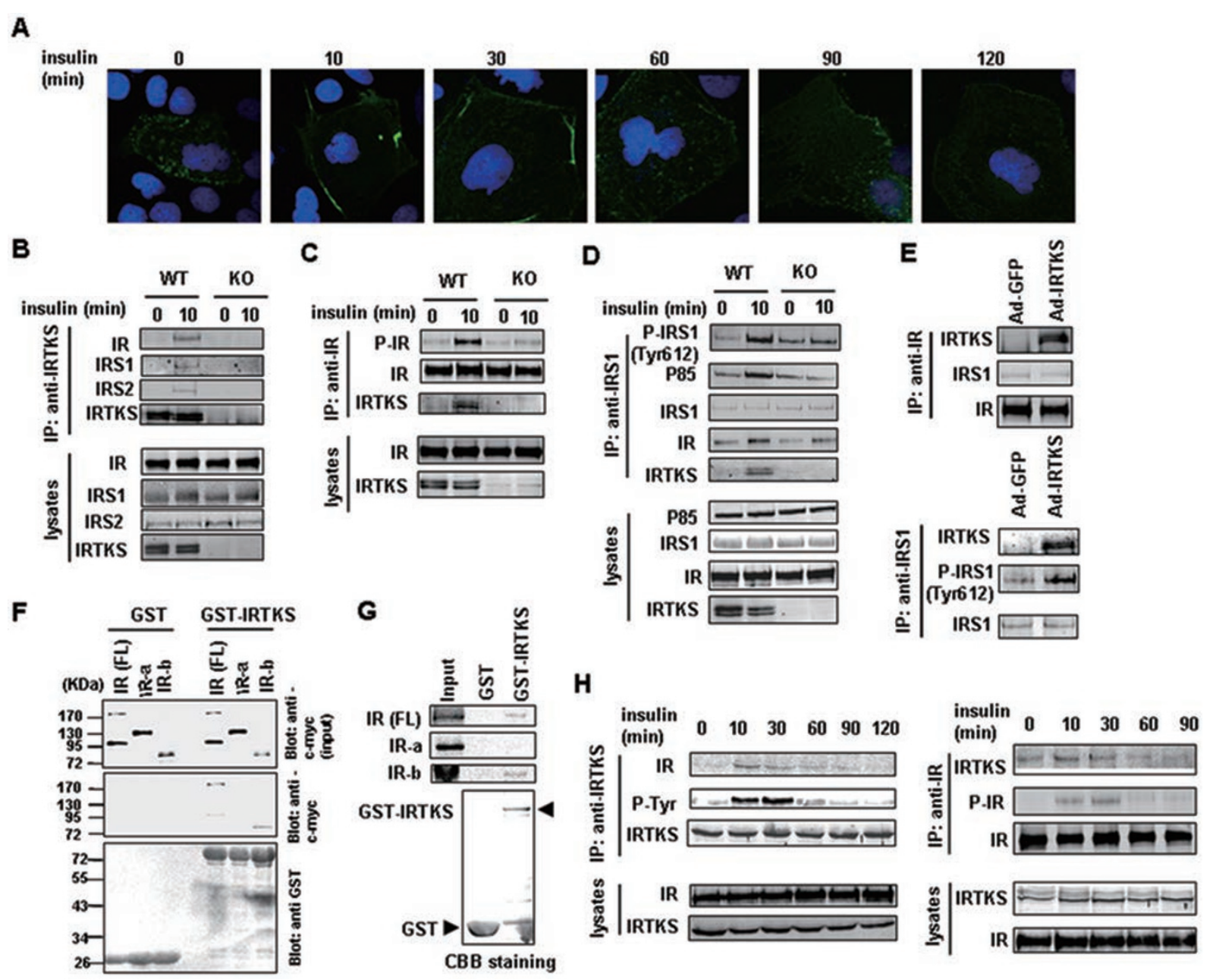

Figure 5 IRTKS binds to insulin receptor and IRS1. (A) Dynamic images of Huh-7 cells transfected with the recombinant expression plasmid containing GFP-tagged IRTKS were shown upon insulin stimulation for the indicated times. (B-D) Co-IP experiments in liver extracts of IRTKS-KO mice and WT littermates with anti-IRTKS, -IR and -IRS1 antibodies, respectively. (E) Co-IP experiments in liver extracts of IRTKS-KO mice infected with Ad-GFP or Ad-IRTKS were performed using anti-IR and -IRS1 antibodies, respectively. (F, G) GST pull-down assays. (F) Glutathione-Sepharose beads coupled with either GST alone or GST-IRTKS were incubated with YY-8103 cell lysates containing myc-tagged full-length (FL) IR, myc-IR $\alpha$ or myc-IR $\beta$ subunits. The positions of the molecular mass markers were shown on the left. (G) In vitro translated ${ }^{35} \mathrm{~S}-\mathrm{labeled} \mathrm{IR}(\mathrm{FL})$, IR $\alpha$ and IR $\beta$ subunits were incubated with purified GST or GST-IRTKS fusion protein coupled with Glutathione-Sepharose beads, respectively. The bound proteins were subjected to SDS-PAGE followed by autoradiography. Coomassie brilliant blue (CBB) staining on SDS-PAGE showed the GST and GST-IRTKS proteins. (H) Co-IP experiments with anti-IRTKS or -IR antibodies were performed in liver extracts of C57BL/6 mice injected with insulin $(1 \mathrm{U} / \mathrm{kg})$ for the indicated times. Western blotting assay was employed to assess the dynamic change of association of IRTKS with IR.

teract with IR. The data revealed that IRTKS physically interacts with IR $\beta$ chain, but not the IR $\alpha$ chain (Figure $5 \mathrm{~F}$ and $5 \mathrm{G})$. Analysis with normal IgG as a negative control is shown in Supplementary information, Figure S6E. These data collectively indicate that IRTKS functions as an adaptor of IR and positively regulates insulin signaling.

We noted that, in the presence of insulin stimulation,
IRTKS tyrosine phosphorylation and the association of IRTKS with IR were enhanced (Figure 5B and 5C, Supplementary information, Figure S6A). We further evaluated the dynamic changes in the association between IRTKS and IR induced by insulin via a reciprocal Co-IP assay. The association between both proteins was enhanced after insulin stimulation and reached a peak at 10-30 min (Figure 5H and Supplementary information, 
Figure S6F), in consistent with the dynamic localization of GFP-tagged IRTKS upon insulin stimulation in Huh-7 cells (Figure 5A).

Together, these data indicate that IRTKS could modulate IR tyrosine phosphorylation and IR-IRS1signaling by acting as an adaptor of IR whereas IRTKS deficiency could attenuate insulin-induced IR phosphorylation and downstream signaling, thereby leading to insulin resistance.

\section{IRTKS levels are decreased in diabetic mice}

To investigate the role of IRTKS in the pathogenesis of type 2 diabetes mellitus (T2DM), we first evaluated the expression of IRTKS in diabetic $d b / d b$ mice. IRTKS protein and mRNA levels were significantly reduced in the liver and adipose tissue of diabetic $d b / d b$ mice compared with lean littermates (Figure 6A and 6B, Supple- mentary information, Figure S7A). This result implies that downregulation of IRTKS could be associated with diabetes development. As excess caloric intake could contribute to the development of T2DM, we evaluated IRTKS protein and mRNA levels in the liver and adipose tissue of high-fat diet (HFD)-induced obese mice with insulin resistance. IRTKS expression was significantly decreased in the liver and adipose tissue of obese mice (Figure 6C and 6D, Supplementary information, Figure $\mathrm{S} 7 \mathrm{~A})$. We also evaluated the expression of IRTKS in livers from patients with T2DM by real-time RT-PCR. IRTKS mRNA levels in these livers were also significantly reduced (Supplementary information, Figure S7B). These data suggest that IRTKS deficiency could be crucial for insulin resistance and the development of T2DM.

Lifestyle affects gene expression via epigenetic mechanisms [16]. To address the epigenetic mechanism
A
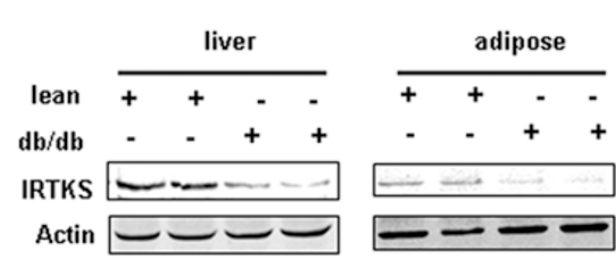

C
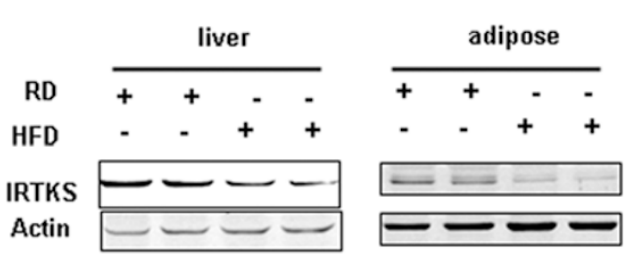

E

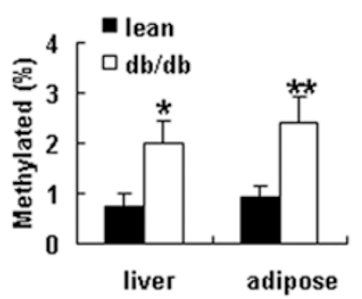

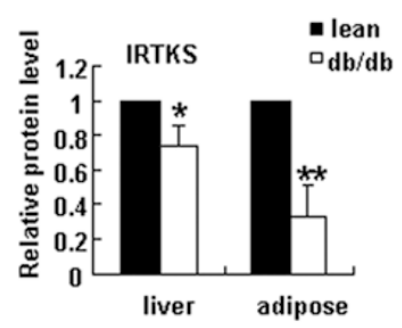

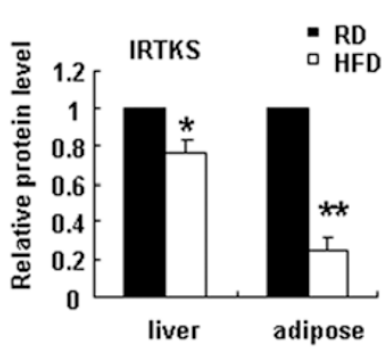

B

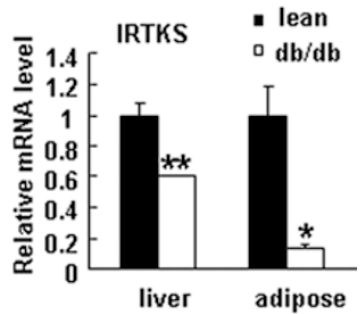

D

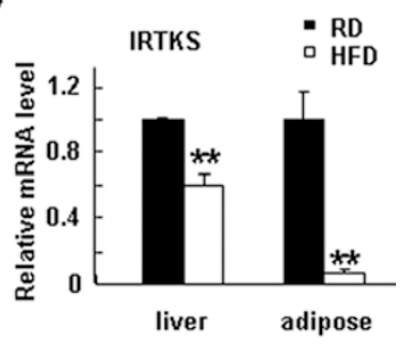

Figure 6 IRTKS downregulation and DNA hypermethylation in diabetic mice. (A, B) IRTKS expression in liver and adipose tissue of $d b / d b$ mice and lean mice ( $n=5$ or 6$)$ determined by western blotting (A) and real-time PCR (B). (C, D) IRTKS expression in liver and adipose tissue of C57BL/6 mice fed with a high-fat diet (HFD) and regular diet (RD) $(n=5)$ determined by western blotting (C) and real-time PCR (D). (E, F) DNA methylation levels of the $\mathrm{CpG}$ island near the transcription start site (TSS) of IRTKS in livers and adipose tissue from $d b / d b$ mice $(n=3)(E)$ and HFD-induced obese mice $(n=2)(F)$. Data are presented as the mean \pm sem. ${ }^{*} P<0.05,{ }^{* *} P<0.01$ versus control. 
responsible for IRTKS downregulation in diabetes and obesity, we analyzed the regulatory elements of the human and mouse IRTKS gene. Interestingly, there are two $\mathrm{CpG}$ islands in the gene. One island is near the transcription start site (TSS) and shares high nucleotide identity between human and mouse IRTKS (Supplementary information, Figure S8A and S8B). We proposed that high DNA methylation of the $\mathrm{CpG}$ island could be associated with low IRTKS expression in diabetes and HFD-induced obesity. To test this hypothesis, we evaluated the DNA methylation status of the CpG island near the TSS in diabetic patients and mice by bisulphite-treatment DNA sequencing. The DNA methylation levels of the $\mathrm{CpG}$ island were significantly elevated in the livers and adipose tissue of $d b / d b$, HFD mice and diabetic patients, compared with controls (Figure 6E and 6F, Supplementary information, Figure S8C), which implies that reduced IRTKS expression could be ascribed to DNA hypermethylation of the $\mathrm{CpG}$ island in diabetic and obese subjects.

\section{Discussion}

Members of the IRSp53/MIM homology domain family are subdivided into two groups: the IRSp53-like members and the MIM-like members [3, 4]. IRSp53like proteins include IRSp53, IRTKS and BAIAP2L2 (FLJ22582), while the MIM-like proteins include two members, MIM and ABBA. All members of IRSp53/ MIM homology domain family have been shown to play an important role in the formation of plasma membrane protrusions $[2,4,5]$. It was recently reported that BAIAP2L2 is involved in clathrin-mediated endocytosis [17]. MIM has been shown to be involved in cancer invasion and metastasis [18]. Similar to IRSp53, IRTKS plays an important role in the formation of membrane protrusions [10, 19]. It should be noted that although IRSp53 and IRTKS were known to be tyrosinephosphorylated in response to insulin stimulation [6, $10]$, very little is known about the role of these proteins in insulin signaling and resistance and the development of diabetes. IRSp53-deficient mice demonstrated cognitive deficits but not insulin resistance and diabetes. However, unlike IRSp53, which is highly expressed in the brain, IRTKS has a widespread tissue distribution, especially in liver [10]. This study revealed that IRTKS is a component of insulin signaling and is required for glucose homeostasis.

In insulin signal transduction, insulin binds to membrane IR, which is a tyrosine kinase that then undergoes autophosphorylation and catalyzes the phosphorylation of downstream intracellular proteins, including the key substrates IRS1 and IRS2, which act as the docking proteins that link IR with other signaling molecules such as p85, a regulatory subunit of PI3K. PI3K-AKT signaling regulates glucose and lipid metabolism. Previous studies of monogenic KO mice targeting key components of insulin signaling pathway demonstrated that only mice lacking IR or IRS1/2 exhibited insulin resistance, whereas knockouts of individual isoforms, such as p85, of the regulatory subunit of PI3K were paradoxically more insulin sensitive than normal mice [20-22]. In the present work, we show that IRTKS functions as an IR adaptor and is required for the activation of the IR-IRS1-AKT signaling pathway via tyrosine phosphorylation. IRTKS deficiency resulted in decreased phosphorylation of IR, IRS1 and $\mathrm{AKT}$, which could be responsible for insulin resistance. Hepatic insulin resistance leads to the elevated serum glucose level in IRTKS-KO mice, and certainly, we cannot rule out the possibility that altered insulin signaling in other tissues such as adipose tissue could also contribute to the phenotype, although the ectopic expression of IRTKS in liver via adenovirus could largely recover the fasting glucose level and insulin sensitivity in IRTKS-KO mice.

In this study, female IRTKS-KO mice displayed a mild insulin resistance compared with male KO mice, implying that the gender-related differences could be involved in IRTKS-deficient mice. Premenopausal women have a lower prevalence of obesity and type 2 diabetes mellitus as compared to age-matched men, while the prevalence of T2DM in postmenopausal women was significantly increased than premenopausal women. Estrogen participates in the modulation of insulin sensitivity, energy balance, and body composition $[23,24]$. The lack of a robust phenotype in female mice in this study may also be related to the differences of sexual hormones between them. In this study, we examined the IRTKS mRNA level in the livers from diabetic patients. IRTKS mRNA level was significantly reduced in male patients (Supplementary information, Figure S7B), and exhibited the decreasing trend in the limited female cases, only four patients, despite no significant difference. To further determine the effect of hepatic IRTKS on female patients, we should examine the IRTKS mRNA level in the livers from more female subjects in the future. In addition, the percentage of body fat is often greater in type 2 diabetic females compared with that in male subjects [25]. Therefore, we also should examine the expression level of IRTKS in adipose tissue from type 2 diabetic females in further investigation. These observations will be necessary for evaluating the role of IRTKS in female patients.

The epigenetic mechanisms could provide a molecular link between diabetes and environmental factors, including diet $[26,27]$. Recent studies have shown that 
the promoters of certain genes, including leptin [28], peroxisome proliferator activated receptor $\gamma$, hepatic glucokinase and L-type pyruvate kinase [30], were significantly hypermethylated in HFD-induced obese animals. Interestingly, in this study, in addition to diabetic patients and $d b / d b$ mice, HFD-induced insulinresistant mice also exhibited DNA hypermethylation of IRTKS promoter (Figure 6), which suggests that extra food intake could be associated with the epigenetic events, although the underlying molecular mechanism remains unknown. Increasing IRTKS expression by epigenetic modifications might be a promising strategy to treat T2DM in the future.

\section{Materials and Methods}

\section{Mice}

IRTKS-KO ES cells were obtained from Shanghai Bimodal Organism Science \& Technology Development Co. Ltd.. These ES cells contain an entire deletion of exon 1 of IRTKS, which is replaced by a $2.0-\mathrm{kb} \mathrm{PGK} / \mathrm{Neo}$ cassette in the IRTKS targeting vector. The primer for the genetic identification: $5 \mathrm{~L}, 5^{\prime}$-TCG CCG GCC AGT CTA GTC AAG TCA GG-3'; Neo-R, 5'-CTG AGC CCA GAA AGC GAA GGA-3'; Neo-F, 5'-CCT CCC CCG TGC CTT CCT TGA C-3'; 3R, 5'-CCG AGG GCA AAT TCT GGG CAC ACT AT-3'. IRTKS-KO mice were generated by Shanghai Bimodal Organism Science \& Technology Development Co. Ltd. We backcrossed IRTKS KO mice onto a C57BL/6 background for more than 6 generations. Mice were housed in a 12-h light, 12-h dark cycle. All other mice were from Shanghai Laboratory Animal Center, Chinese Academy of Sciences. Mice were fed with a regular diet (RD) and had free access to water and diet.

\section{Hyperinsulinemic-euglycemic clamp studies}

Clamps were performed on 20 -week-old male mice weighing 30 g. An indwelling catheter for infusion of insulin and glucose was placed into the left cervical vein under diethyl ether anesthesia. Mice were allowed to recover for 5-7 days, until they had regained $95 \%-100 \%$ of their initial body weight. After a $12 \mathrm{~h}$ fast, a 180 min hyperinsulinaemic-euglycaemic clamp study was conducted in awake, freely moving mice. Insulin was infused at 2.5 $\mathrm{mU} / \mathrm{kg} \cdot \mathrm{min}$.

\section{GTT and ITT}

GTT and ITT were performed by intraperitoneal injection of glucose $(1.5 \mathrm{~g} / \mathrm{kg})$ after fasting for $6 \mathrm{~h}$ or of insulin (1 unit/ $\mathrm{kg}$ ) without fasting. Blood glucose levels were measured before injection and at 15, 30, 60, 90 and 120 min after injection.

\section{Recombinant adenovirus vector and administration}

Plasmid pShuttle-CMV-IRTKS-IRES-hrGFP was constructed, and then IRTKS-IRES-hrGFP element was subcloned into AdEasy XL Adenoviral Vector System (Stratagene) (Ad-IRTKS). Ad-GFP was used as control. Twenty-week old WT and IRTKS-KO mice and 8-week-old $d b / d b$ mice were injected with these adenovirus vectors $\left(1 \times 10^{9} \mathrm{pfu}\right)$ via tail vein. GTTs and ITTs were conducted at 7-8 days after adenovirus injection. Mice were killed 14 days after the adenovirus injection.

\section{Antibodies}

Anti-IRTKS rabbit polyclonal antibody was produced by our lab as used in our published paper [15]. Briefly, the homemade rabbit polyclonal anti-IRTKS antibody was raised against the recombinant GST-IRTKS fusion protein containing whole IRTKS. Anti-actin and anti-pIR $\beta$ (Tyr1162/1163) antibodies were from Santa Cruz Biotechnology. Anti-pAKT (Ser473), anti-AKT, antipGSK3 $\beta$ (S9), anti-GSK3 $\beta$, anti-IR $\beta$, anti-IRS1, anti-IRS2, antiubiquitin and anti-phosphorylated Tyr antibodies were obtained from Cell Signaling. Anti-pIRS1 (Tyr612) and anti-GFP antibodies were from Abcam. The Rat/Mouse Insulin ELISA Kit was from Linco Research.

\section{Immunoprecipitation assay}

The $1 \mathrm{mg}$ of liver or cell lysates were incubated with the indicated antibodies at $4{ }^{\circ} \mathrm{C}$ overnight, and then incubated with protein $\mathrm{G}$ sepharose beads at $4{ }^{\circ} \mathrm{C}$ for another $4 \mathrm{~h}$. Each immunoprecipitate was washed four times with lysis buffer and then analyzed by immunoblotting.

\section{siRNA synthesis}

Two siRNAs against IRTKS were designed and chemically synthesized by Shanghai GenePharma Co. siRNA-1 (sense, 5'CCA GUC CCU UGA UCG AUA UTT-3' and anti-sense, 5'-AUA UCG AUC AAG GGA CUG GTA-3'); siRNA-2 (sense, 5'-GCU UAA GCA AAU CAU GCU UTT-3' and anti-sense, 5'-AAG CAU GAU UUG CUU AAG CAG-3').

\section{MEFS}

Mouse MEFs were isolated from E13.5 mouse embryos. IRTKS-KO MEF cells were infected with adenovirus at $1 \times 10^{8}$ $\mathrm{pfu} / \mathrm{ml}$.

\section{Immunofluorescence}

An immunofluorescence assay was performed to detect dynamic localization of GFP-tagged IRTKS in Huh7 cells. Cells were fixed in $4 \%$ paraformaldehyde and permeabilized with $0.1 \%$ $(\mathrm{v} / \mathrm{v})$ Triton X-100/PBS. Slides were incubated with blocking buffer (5\% horse serum in PBS) for $2 \mathrm{~h}$ at room temperature. After rinsing, the slides were analyzed using immunofluorescence microscopy.

\section{GST pull-down assay}

Plasmids containing IR (full length), IR $\alpha$ chain, IR $\beta$ chain and IRS-1 constructs were transcribed and translated in vitro with the TNT System (Promega). Resultant ${ }^{35} \mathrm{~S}$-labeled products and $10 \mu \mathrm{g}$ of GST immobilized derivatives were incubated at $4{ }^{\circ} \mathrm{C}$ in $500 \mu \mathrm{l}$ binding buffer $(50 \mathrm{mM}$ Tris- $\mathrm{HCl}, \mathrm{pH} 7.5,150 \mathrm{mM} \mathrm{NaCl}$, $1 \mathrm{mM}$ EDTA, $0.3 \mathrm{mM}$ DTT, $0.1 \% \mathrm{NP}-40$ and protease inhibitor cocktail (Sigma)). Precipitated beads were washed four times with binding buffer, followed by protein elution in SDS-PAGE sample buffer, analysis by SDS-PAGE, and radioactive detection by a PhosphorImager (Amersham).

\section{Bisulfite DNA sequencing}

Bisulfite treatment was performed as previously described [31]. Briefly, modified DNA (20 ng) was amplified by PCR with 
Hotstart Taq (Qiagene) and primers specific to respective gene promoters. PCR products were gel-extracted and subcloned into pMD19-T vectors (TaKaRa, Japan) for DNA sequencing (BGI, Shanghai, China).

\section{Statistical analysis}

Unless otherwise specified, all bars show standard deviation. Significance was calculated using student's $t$-test $\left({ }^{*} P<0.05\right.$; ${ }^{* *} P$ $\left.<0.01 ;{ }^{* * *} P<0.001\right)$.

\section{Acknowledgments}

We gratefully thank Lun-Xiu Qin (Liver Cancer Institute, Medical Center of Fudan University) for providing clinical samples. We also gratefully acknowledge support from the National Key Basic Research Program of China (2010CB529200), the National Natural Science Foundation of China (81071722 and 81272271), and the China National Key Projects for Infectious Disease (2012ZX10002012-008 and 2013ZX10002010-006).

\section{References}

1 Hu RM, Han ZG, Song HD, et al. Gene expression profiling in the human hypothalamus-pituitary-adrenal axis and full-length cDNA cloning. Proc Natl Acad Sci USA 2000; 97:9543-9548.

2 Ahmed S, Goh WI, Bu W. I-BAR domains, IRSp53 and filopodium formation. Semin Cell Dev Biol 2010; 21:350-356.

3 Scita G, Confalonieri S, Lappalainen P, Suetsugu S. IRSp53: crossing the road of membrane and actin dynamics in the formation of membrane protrusions. Trends Cell Biol 2008; 18:52-60.

4 Zhao H, Pykäläinen A, Lappalainen P. I-BAR domain proteins: linking actin and plasma membrane dynamics. Curr Opin Cell Biol 2011; 23:14-21.

5 Suetsugu S, Toyooka K, Senju Y. Subcellular membrane curvature mediated by the BAR domain superfamily proteins. Semin Cell Dev Biol 2010; 21:340-349.

6 Yeh TC, Ogawa W, Danielsen AG, Roth RA. Characterization and cloning of a $58 / 53-\mathrm{kDa}$ substrate of the insulin receptor tyrosine kinase. J Biol Chem 1996; 271:2921-2928.

7 Heung MY, Visegrady B, Fütterer K, Machesky LM. Identification of the insulin-responsive tyrosine phosphorylation sites on IRSp53. Eur J Cell Bio 2008; 87:699-708.

8 Sawallisch C, Berhörster K, Disanza A, et al. The insulin receptor substrate of $53 \mathrm{kDa}$ (IRSp53) limits hippocampal synaptic plasticity. J Biol Chem 2009; 284:9225-9236.

9 Kim MH, Choi J, Yang J, et al. Enhanced NMDA receptormediated synaptic transmission, enhanced long-term potentiation, and impaired learning and memory in mice lacking IRSp53. J Neurosci 2009; 29:1586-1595.

10 Millard TH, Dawson J, Machesky LM. Characterisation of IRTKS, a novel IRSp53/MIM family actin regulator with distinct filament bundling properties. J Cell Sci 2007; 120( Pt 9):1663-1672.

11 Vingadassalom D, Kazlauskas A, Skehan B, et al. Insulin receptor tyrosine kinase substrate links the E. coli $\mathrm{O} 157: \mathrm{H7}$ actin assembly effectors Tir and $\operatorname{EspF}(\mathrm{U})$ during pedestal formation. Proc Natl Acad Sci USA 2009; 106:6754-6759.
12 Aitio O, Hellman M, Kazlauskas A, et al. Recognition of tandem PxxP motifs as a unique Src homology 3-binding mode triggers pathogen-driven actin assembly. Proc Natl Acad Sci USA 2010; 107:21743-21748.

13 Crepin VF, Girard F, Schüller S, Phillips AD, Mousnier A, Frankel G. Dissecting the role of the Tir:Nck and Tir:IRTKS/ IRSp53 signalling pathways in vivo. Mol Microbiol 2010; 75:308-323.

14 Youngren JF. Regulation of insulin receptor function. Cell Mol Life Sci 2007; 64:873-891.

15 Wang KS, Chen G, Shen HL, et al. Insulin receptor tyrosine kinase substrate enhances low levels of MDM2-mediated p53 ubiquitination. PLoS One 2011; 6:e23571.

16 Gluckman PD, Hanson MA, Buklijas T, Low FM, Beedle AS. Epigenetic mechanisms that underpin metabolic and cardiovascular diseases. Nat Rev Endocrinol 2009; 5:401-408.

17 Veltman DM, Auciello G, Spence HJ, Machesky LM, Rappoport JZ, Insall RH. Functional analysis of Dictyostelium IBARa reveals a conserved role of the I-BAR domain in endocytosis. Biochem J 2011; 436:45-52.

18 Xie F, Ye L, Ta M, Zhang L, Jiang WG. MTSS1: a multifunctional protein and its role in cancer invasion and metastasis. Front Biosci (Schol Ed) 2011; 3:621-631.

19 Saarikangas J, Zhao H, Pykäläinen A, et al. Molecular mechanisms of membrane deformation by I-BAR domain proteins. Curr Biol 2009; 19:95-107.

20 Biddinger SB, Kahn CR. From mice to men: insights into the insulin resistance syndromes. Annu Rev Physiol 2006; 68:123158.

21 Pattaranit R, van den Berg HA, Spanswick D. The development of insulin resistance in Type 2 diabetes: insights from knockout studies. Sci Prog 2008; 91(Pt3):285-316.

22 Saltiel AR, Kahn CR. Insulin signalling and the regulation of glucose and lipid metabolism. Nature 2001; 414:799-806.

23 Szmuilowicz ED, Stuenkel CA, Seely EW. Influence of menopause on diabetes and diabetes risk. Nat Rev Endocrinol 2009; 5:553-558.

24 Manrique C, Lastra G, Habibi J, Mugerfeld I, Garro M, Sowers JR. Loss of estrogen receptor $\alpha$ signaling leads to insulin resistance and obesity in young and adult female mice. Cardiorenal Med 2012; 2:200-210.

25 Miyazaki Y, Glass L, Triplitt C, Wajcberg E, Mandarino LJ, DeFronzo RA. Abdominal fat distribution and peripheral and hepatic insulin resistance in type 2 diabetes mellitus. $\mathrm{Am} \mathrm{J}$ Physiol Endocrinol Metab 2002; 283:E1135-E1143.

26 Ling C, Groop L. Epigenetics: a molecular link between environmental factors and type 2 diabetes. Diabetes 2009; 58:2718-2725.

27 Ng SF, Lin RC, Laybutt DR, Barres R, Owens JA, Morris MJ. Chronic high-fat diet in fathers programs $\beta$-cell dysfunction in female rat offspring. Nature 2010; 467:963-966.

28 Milagro FI, Campión J, García-Díaz DF, Goyenechea E, Paternain L, Martínez JA. High fat diet-induced obesity modifies the methylation pattern of leptin promoter in rats. J Physiol Biochem 2009; 65:1-9.

29 Fujiki K, Kano F, Shiota K, Murata M. Expression of the peroxisome proliferator activated receptor gamma gene is repressed by DNA methylation in visceral adipose tissue of mouse models of diabetes. BMC Biol 2009; 7:38. 
30 Jiang M, Zhang Y, Liu M, et al. Hypermethylation of hepatic glucokinase and L-type pyruvate kinase promoters in high-fat diet-induced obese rats. Endocrinology 2011; 152:1284-1289.

31 Herman JG, Graff JR, Myöhänen S, Nelkin BD, Baylin SB.
Methylation-specific PCR: a novel PCR assay for methylation status of CpG islands. Proc Natl Acad Sci USA 1996; 93:9821-9826.

(Supplementary information is linked to the online version of the paper on the Cell Research website.) 